\title{
The Effects of Power Relations on the Curriculum Experiences of French-Speaking Students in a Private Institution's Academic Community of Practice
}

\author{
C.A. Adebanji \\ College of Education, University of South Africa, Pretoria, South Africa \\ dadebanji2005@gmail.com \\ M.T. Gumbo \\ College of Education, University of South Africa, Pretoria, South Africa \\ gumbomt@unisa.ac.za
}

\author{
Doi:10.5901/mjss.2014.v5n20p1233
}

\begin{abstract}
This paper reports on the findings of a study in which we explored power relations and their effects on the curriculum experiences of French-speaking students in an Academic Community of Practice (ACOP) at a private tertiary institution. French-speaking students (FSS) negotiated the rigours of higher education as experienced by the other students in the midst of institutionalized power relations which limited their capacity to effectively integrate into the ACOP. A prominent consequence of linguistic power relations among French-speaking students (FSS) was a delayed transition from French to English. This was as a result of the monopoly of indigenous languages employed by South African students. Utilising a case study approach and narrative inquiry it was found that FSS had to grapple with the effects of linguistic power relations, financial power relations, inflationary power relations and workplace power relations. Lave and Wenger (1991, 115-116) present a framework on which our findings are premised because their work evolved the "continuity-displacement contradiction" in communities of practice. We found that issues of the "continuity-displacement contradiction" were subdued with elapsed time as suggested by Lave and Wenger (1991) because those affected were continuously displaced from the terrains of relevance.
\end{abstract}

Keywords: Continuity-displacement contradiction, power relations, workplace curriculum, linguistic transition, Academic Communities of Practice (ACOP)

\section{Introduction}

In a typical academic institution there are roles and responsibilities expected to be in place among stakeholders. These roles and responsibilities are institutionalised due to certain basic principles to foster teaching and learning. The role of a facilitator of learning is to ensure that learning takes place among students. Students on the other hand are charged with the awareness to take a responsibility for their learning. In the midst of these responsibilities, there are certain forces which may tend to create insurgence in terms of roles and responsibilities among stakeholders in an ACOP. Academic communities of practice, drawing on Otten (2009), comprise lecturers, students, academic managers, student advisors and deans of faculties charged with different responsibilities in an academic institution. French-speaking students came to study in South Africa in a multiculturally diverse milieu which predisposed them to negotiating a number of experiences. They are required to negotiate a transition from French to English in the midst of several other languages used to communicate informally at the ACOP. The issues regarding payment of fees are important because of the advent of power relations among stakeholders in an ACOP, drawing on Lave and Wenger (1991). Studying in a novel multicultural milieu could give rise to power dynamics, capable of impacting on the curriculum experiences of nonEnglish-speaking students (specifically FSS in this study) because of the requirement to deal with intercultural adjustments. Since FSS were situated in a novel multicultural milieu, it becomes important to utilise the theories underpinning situated leaning (e.g., Legitimate peripheral participation (LPP) and the communities of practice (COP) framework) to explore the issues of power relations that may ensue between them and the other stakeholders in the ACOP.

We define stakeholders in an ACOP as the FSS, lecturers, tutors, academic managers, student advisors and the top management in the ACOP, to mention but a few. We explore power relations by employing the Community of Practice (COP) framework where novices are granted permission to belong to the community via Legitimate Peripheral 
Participation (LPP) as a result of certain eligibility factors, drawing on Lave and Wenger (1991). Lave and Wenger institutionalised the LPP theory, indicating that on the periphery of a typical COP, beginners commence a series of negotiations by legitimately and peripherally participating in the culture of the community in a centripetal manner until full participation is achieved. Although the FSS had to negotiate a number of experiences as it was the case with South African students and the other foreign students from other countries, we were interested in how the FSS navigated the terrains of higher education because they had to adjust to learning English, adapt to a new didactic culture and acculturate to the mainstream society within the limited time they had to stay in South Africa.

We draw on Lave's and Wenger's $(1991,116)$ scholarship that the idea of granting access to beginners with their own standpoints to communities of practice is responsible for "all the tensions of the continuity-displacement contradiction." The "continuity-displacement contradiction" dynamics is taken as the struggle for survival and exertion of authority to declare and attain an identity for recognition among stakeholders. We take on the suggestions of Lave and Wenger (1991) by applying this phenomenon to the ACOP being a multicultural milieu. These contradictions appear during the stage of traineeship between beginners and experts in a COP. At this transitional stage the disparate avenues through which experts and novices construct and sustain their uniqueness clash and create conflicting standpoints on the practice and the development thereof (Lave \& Wenger, 1991, 115). Lecturers, mentors, academic managers, student advisors and tutors are experts in the COP because they directly assist students in their day-to-day learning and wellbeing. Consequently we problematize the interaction and association of stakeholders in the ACOP in their dealings with the focus of this study (the French-speaking students).

Lave and Wenger $(1991,115-116)$ posit that as long as the encounters through which the "continuity-displacement contradiction" is displayed pertains to power show, which more often than not is the case - how the conflict is dealt with is assumed to vary as power dynamics fluctuate. This claim predisposes us to using the transactions between the FSS and the other stakeholders in the ACOP to explore avenues evolving in the relations of power among them. Furthermore it allows us to explore how such relations of power are dealt with. In an attempt to unravel the intellectual puzzle we sought answers to the following research question: How do power relations among other stakeholders challenge the curriculum experiences of FSS in the ACOP? The underlying assumption that paved the way for this inquiry is presented: The curriculum experiences of FSS are challenged by power relations in the ACOP. We embarked on this inquiry because minimal attention has been given to the issues of power relations among stakeholders in the private higher education of South Africa.

The intellectual dilemma is presented in this way. Firstly, we provide a succinct background of the FSS and the research site. Secondly, we set the scene by discussing the findings in literature to situate issues pertaining to power relations in COP and the curriculum. We discuss the COP model to foreground the topic under exploration against instances of power relations in an ACOP. Thirdly, the research strategy is discussed. Fourthly, the findings are presented and discussed by providing narratives obtained from the respondents of the study to corroborate claims made in the study and in the voluminous literature. Finally, conclusion and recommendations are drawn in relation to the emergence, consequence and amelioration of power relations in an ACOP.

\section{Background Context}

The research site, pseudonamed in this study as Westville University, was located within the Gauteng Province, accessible to many students who at the time of investigation either lived at the hostel located adjacent to the main campus, or travelled by road from neighbouring cities and towns to the academic institution. The context of this study involved an exploration of the factors impacting upon the curriculum experiences of FSS in a private tertiary institution. In conducting this study the campus environment, lecturers, facilities available for higher education, language policy, as well as issues relating to the acculturation and identity negotiation of the students from French-speaking countries were explored. The international students' office at the university/college campus also offered a rich source of data for the study (otherwise known as the student advisory unit). These students were from the Democratic Republic of Congo (DRC), Cameroon, Gabon, Republic of Chad and Benin Republic. They came to study in South Africa because they wanted to become bilingual to stand the chance of partaking in a globalized world.

\section{Unravelling the Scholarly Dilemma: Power Relations in ACOP}

It is challenging to discuss issues of power relations in the context of this study without exploring the LPP model, COP framework and issues bordering on curriculum experiences because these experiences denote scenarios operating in situated learning experiences. Furthermore Lave and Wenger (1991) made suggestions about the "continuity- 
displacement contradiction" dynamics under situated learning experiences. Situated learning is encompassed by a general description known as "situativity theory" (Durning and Artino, 2011). According to Durning and Artino $(2011,188)$ :

\begin{abstract}
... situativity theory stresses the social nature of cognition, meaning, and learning, with emphasis on the importance of the participants and the environment, as well as the evolving interaction between the participants and the environment within which thinking and learning occur
\end{abstract}

The concept of LPP was originally employed by Lave and Wenger (1991) in their exploration of apprenticeship as a representation of situated scholarship. The LPP model is seen in this paper as a point of convergence for the COP scholarship because it announces the arrival of new entrants into the communities of practice. Communities of Practice comprise groups of individuals who share a concern or a craving for what they do and who work together frequently to gain expertise (Wenger, 1998). Consequently, issues of power dynamics are envisaged where membership operates as the modus operandi.

This framework predisposes this study to exploring the effects that may ensue from the FSS' encounters with the other stakeholders. We therefore perceive Westville University as a bigger COP, with subsets of other little COP in association to attaining an identity. Consequently, practice becomes the vehicle through which the domain would be accomplished - an enterprise which is seen as capable of evolving relations of power among participants. We use this same principle to extrapolate other salient requirements of the FSS, such as the need to acculturate and define their identities, all of which involve power relations with stakeholders. We perceive that the FSS are required to acculturate to the ACOP for learning to ensue. Acculturation is conceptualised as a curriculum experience - taken as a conscious effort to negotiate a usual culture with a novel one, drawing on Yeh et al. (2008). Utilizing the terminology of Wenger (1998, 108), the movement of FSS from one subset of COP to another consolidates practice, referred to as "brokering" or negotiation to reach the domain. Similarly, to attain a set identity, "brokering" is essential, conceptualized to follow the same identified route of vacillating between COP in a bigger ACOP. In order for the FSS to attain academic identity, they are assumed to have to move between subsets of COP in a bigger ACOP. Academic identity in this context involves the development of the necessary academic self-esteem, sufficient to take responsibility for their learning, drawing on Vandeyar (2010). As they take responsibility to attend lectures, tutorials, seek consultation with lecturers, they are perceived as engaging in what Wenger $(1998,108)$ refers to as "brokering" to attain an identity. These interspersed movements are indicative of participants moving from one subset of COP to the other, conceptualized to generate trickles of power relations to attain a set identity. To this end, the brokering of FSS with the other stakeholders is presented as a predicament in this paper.

The language of communication is vital for meaning negotiation in an ACOP, and the development of academic identity. According to Chow (2006), it was reported that the language of communication is capable of disentangling the learning curriculum. The learning of concepts within the community by FSS therefore depends on their ability to understand curriculum via the appropriate terrain of language. A predominant use of indigenous languages on campus is predicted to delay the transition of FSS to English. When there is persistence in the use of indigenous languages in the academic institution, marginalization of the language of instruction may ensue because interaction among stakeholders could be restricted. When interaction is restricted, it is predicted that the ability of FSS to participate actively in subsets of COP may be hampered. The issues raised in this review of the voluminous literature are capable of sustaining or negating the quintessence of survival among the FSS of this study in the midst of institutional stakeholders. Consequently, an exploration to reach a submission in terms of the power dynamics experienced by FSS in the midst of South African students and other international students was empirically conducted.

\title{
4. The Research Strategy
}

In this study we took on the role of an interpretivist, bordered by the constructivist line of reasoning which perceives reality in a unique perspective. This is in agreement with Burden $(1997,242)$, that one should use novel perceptions of judgment and documentation, competent at communicating expressively to new and intricate avenues of insight. We comprehended how stakeholders obtained, appraised, and substantiated knowledge and what they regarded as knowledge construction on the issues of power relations. This placed a measure of responsibility on us because we were interested in obtaining information from the FSS and other respondents of the study.

It follows that this study adopted a qualitative route, explored as "a situated activity that locates the observer in the world" of other people (Denzin \& Lincoln, 2005, 3). We took advantage of59 the scholarship of Denzin and Lincoln that qualitative research comprises an assortment of interpretive, logical practices that make the globe perceptible to explore 
this research study $(2005,3)$, by delving into the issues pertaining to the curriculum of study and the consequence of interactional perspectives between the stakeholders in the ACOP. A total of 28 FSS were purposively drawn from the predegree programme to the third-year degree programme and interviewed on issues cutting across the curriculum. Out of the 28 FSS interviewed, 15 were interviewed one-on-one while the remaining 13 were interviewed in two sets of focus group sessions between 2012 and 2013. Separate semi-structured interviews were conducted with 5 lecturers who taught them, 5 French-speaking student advisors, the Head of the Foundation Phase Course (pre-degree) and the Dean of the Information Technology (IT) Faculty. To triangulate data, a cohort of 6 South African students (SAS) was interviewed using the focus group technique. The interviews spanned between 30 minutes to an hour.

A combination of content analysis (Mayring, 2000; Sandelowski, 2000) and thematic analysis (Braun and Clarke, 2006) was used to analyze the obtained data. As the transcribed data were carefully perused codes were allocated and repeatedly adjusted as the data evolved more understanding of the research question of the study. This was done to give room for novel data and emerging perceptions concerning the transcribed data (Sandelowski, 2000, 338). This rigorous process was spontaneous and communicating to the extent that it resulted in wide ranging codes and themes. The wide ranging codes were subjected to further analysis to comprehend data associated with issues of power relations in an ACOP, the COP and LPP models and the reviewed literature, drawing on the suggestion of Miles and Huberman (1994).

\title{
5. Findings and Discussion
}

Power relations became obvious from the narratives obtained from many of the stakeholders at Westville University. We present a number of narratives from the FSS and other stakeholders to make claims in this study, suggesting power relations at Westville University. Power relations are discussed under the following headings, namely financial power relations, linguistic/intercultural power relations, inflationary power relations and workplace power relations.

\subsection{Financial power relations}

Issues pertaining to financial power relations were revealed by the FSS, namely Ornella and Candy, to mention but a few out of the 28 FSS interviewed. Ornella was a second-year FSS from Gabon, studying Business Management. Candy was a FSS from the Democratic Republic of the Congo (DRC) studying Biomedicine. Ornella paved the way for an understanding of how financial power relations operate in the ACOP. She owed fees in arrears and was prevented from attending lectures. Although the other students at Westville University were also excluded when they did not pay fees as at when due, the experiences of FSS in this dimension became a challenging one because their sponsors were not within reach to assist them. Secondly, foreign students paid half of their fees upfront before the commencement of tuition. Thirdly FSS were the focus of the study which reflects what the other students at Westville University experienced as well. Ornella's concerns are presented in response to the question put to her in an attempt to explore the challenges confronting her at Westville University:

\begin{abstract}
If you do not pay the school fees as at when due, they block you from coming in to the campus. Even if you have a test, you will not be allowed to write. The institution should try to understand our problems with respect to delays in payment that at times we are challenged in terms of the ease of transferring funds from Gabon to South Africa. If I don't write the tests, then I cannot write exams or go to the next class. This challenge is a stress factor.
\end{abstract}

Ornella's argument centred on the notion that the management of Westville University should allow students to gain access into Westville University instead of preventing them from it when fees are in arrears. It is quite evident that power struggle in terms of the views of management and students is a similitude of the "continuity-displacement contradiction" proposed by Lave and Wenger $(1991,115-116)$ in communities of practice. This issue of financial exclusion challenged their continuity at Westville University by attempting to displace them from concentrating on their studies. An academic manager was approached to discuss this issue of exclusion from tuition when fees were in arrears. She said, "Lecturers have to be paid as at when due, we are compelled to make this issue a policy that students would not have access to tuition when fees are in arrears." The difference in perception between FSS' views and the policy of Westville University in terms of fees in arrears and the requirement of the institution to meet financial obligation to its workforce are seen in this study as power relations between stakeholders.

As far as Candy was concerned, she argued by contending that at times she did not have the $50 \%$ initial payment required for foreign students to start classes. The result of this incapability to meet the demand at Westville University resulted in exclusion from tuition. Evidence is provided in response to the question put to her on the challenges she 
confronted at the school:

You know that this institution is a private university, so the fees are expensive. Sometimes I don't have enough money to pay because a foreign student has to pay $50 \%$ at the beginning of the year, then after that the remaining $50 \%$ has to be paid over a period of 10 months. Sometimes if the $50 \%$ is not available at the beginning of the year, it means I would not be able to start school. At least they know we would eventually pay, so they should not block us from attending lectures.

On the other hand South African students did not have to make an initial payment of $50 \%$ of fees before they were allowed to commence tuition. Westville University made things easy for South African students because they were given the option to pay fees on a monthly basis in equal installments. This was revealed by one of the French-speaking student advisors (Ms. Sammy) who said:

It is more convenient for South African students to pay fees here. They have the privilege of applying for bursary from different establishments. They are allowed to pay monthly compared to foreign students who pay half of the fees at registration. It is not easy for foreign students studying at this institution. The advantage is that French-speaking students are dedicated because they don't want to disappoint their parents.

We argue that this is an emblem of financial power relations because not all the students are given the same treatment at Westville University in terms of the terms and conditions of paying fees. It is true that all of the students whose fees were in arrears were excluded. However the weighting was one-sided because the FSS were under severe pressure in terms of how they were treated on how they paid fees. The facts that South Africa students (SAS) had the privilege of paying fees on a monthly basis and the access to bursary were indications of power dynamics existing between them and the foreign students - indications of the continuity-displacement contradiction in communities of practice as suggested by Lave and Wenger (1991). Although Trimble, Pederson and Rodela (2009) reiterate that the course of attaining intercultural competence takes place with elapsed time, issues pertaining to intercultural competence could be complicated by the dynamics of the "continuity-displacement contradiction" emanating from financial power relations because of the inequalities between foreign and indigenous students. It becomes obvious that to achieve this challenging task in a multicultural milieu, foreign students must be determined, make conscious effort, and imbibe the desire to attain intercultural competence despite that it could be complicated.

Issues pertaining to the "continuity-displacement contradiction" continued unabated throughout the entire duration of this study, suggesting the validity of Lave's and Wenger's (1991) suggestion that they fizzle out with elapsed time because they seemed to have been institutionalised by policy. It thus implies where issues of financial power relations abound in COP, the prevailing force outweighs the lower until a state of suppression is reached to silence the marginalized. The voices of stakeholders who felt the insurgence of power dynamics eventually fizzled out because they did not have any other choice other than to abide by the rules.

\subsection{Linguistic power relations}

According to Ramburuth and Tani (2009), in a multiculturally diverse milieu where different students converge to learn, issues of language barriers abound. They argue that the detrimental consequence of language is not limited to the capacity to learn. It is capable of challenging the confidence to relate with other students. It was found that issues relating to linguistic power relations were not one-sided. Drawing on the scholarly work of Yeh et al. (2008), in a study of Chinese foreign students' cultural interactions, acculturation, family obligations, language use and social support, language and intercultural dynamics are intertwined. Consequently, linguistic power relations and intercultural power relations are interrelated. Both FSS and SAS displayed elements of linguistic power relations. Amanda, a third year FSS studying IT, from Gabon, revealed this experience when she was asked to reflect on her linguistic transitional experience from first year to the third year degree programme. She responded by saying:

One bad habit that I picked is to express myself in French. Before, when I was with French-speaking students, I practised my English. Now, I speak French more often, except when there are other students who do not understand French.

One would expect that FSS would strictly adhere to speaking English at third year level, having stayed at Westville University for such a long period. From the account of Amanda it was not so. According to the observation sessions conducted in this study it was found that FSS minded their enclaves and freely conversed in French with other FSS. This 
practice was found to ostracize other non-FSS from their groups. Similarly, SAS, for example, spoke indigenous languages among themselves, thus excluding the FSS from their groups. Different groups of students accused one another of linguistically ostracizing them from other groups through the adoption of different languages. Resultantly, the extent of association among FSS and indigenous groups was limited. As far as the SAS were concerned the FSS detached from them. SAS felt that the FSS needed to take the initiative to come out of their comfort zones by becoming interested in what mattered to them.

The 6 SAS interviewed in the focus group session agreed that they were willing to interact and associate with the FSS as long as they also became willing to detach from their French-speaking territories to join them. The SAS believed that it was not a matter that pertained to the FSS alone but to all the students. The responses of FSS to questions put to them on issues of the use of English pointed to the subject of self-esteem rather than attending to their requirements to negotiate a transition from French to English. We present their views with respect to the requirements needed to forgo their self-esteem in an attempt to embrace the necessity to interact with the SAS. We present the narratives of Jordan and Jacobus (South African students) as evidence of linguistic power dynamics between FSS and SAS. Jordan's response to the question put to the group in terms of linguistically marginalising the FSS at Westville University is provided:

They must be willing to leave their comfort zone. If they are not willing to leave their comfort zone then I would not also leave because we all have our powers in terms of speaking our own language. I would have to use my power to defend my language so we have to meet halfway at the point of speaking in English.

The response to the question put to the SAS during the focus group interview session met with resistance among them. A question put to them was that FSS claimed that SAS excluded them during discussion sessions. Jordan's response indicated the power embedded in the language of communication. He said, "Everybody has the power to defend his language." This was an indication of power relations that could emanate from the use of a particular language of dominance.

Jacobus responded in the following way, which revealed that issues pertaining to linguistic power relations require consciously taking strides against the latent power dominating language in an ACOP. He mentioned that rejection could ensue if people do not have the courage to break the tide that language offers:

If I go into the midst of the French-speaking students, I would feel uncomfortable at the beginning because we are all afraid of rejection. I am sure they feel the same way when they see us speaking in Zulu. The way friendship is built is that we meet ourselves through other friends. It is not just easy to bump into peoples' groups when they are conversing in their language. It is more than that. There has to be instances of connection to participate in other language speakers' conversations.

Feeling uncomfortable when other language speakers are communicating presents the possibility that power relations operates when a dominant language is used among people. The fact that it takes courage to barge into peoples' linguistic domain suggests that language is laden with power and control. We assert that issues of linguistic power relations pertain to the portrayal of self-esteem among actors in communities of practice where interaction is the key proponent of learning. Although the initiative to overcome low self-esteem proposed by SAS in terms of the FSS associating with them was not institutionalised at Westville University as it was, in the study conducted by Quintrell and Westwood (1994) who confirmed the attendant opportunities involved in a peer pairing initiative that such initiatives could break barriers of linguistic power relations among the stakeholders. The narratives of FSS are considered to reveal issues of linguistic power dynamics in relation to their perception of SAS.

These narratives support our prediction that the use of indigenous languages on campus is capable of delaying the transition of FSS to English. It also limited the capacity of SAS' capability to master English because they also indulged in speaking indigenous languages at Westville University. The experiences of FSS were found to support the declaration of Chow $(2006,109)$, that proficiency in English "is required for communication and for the acquisition of information about the new society." We argue that the communication in a commonly agreed language in an ACOP is capable of ameliorating the effects of linguistic power relations in a multiculturally diverse institution. It facilitates the interaction of stakeholders in an ACOP with one another. As noted by Chow $(2006,109)$, proficiency in English was observed to determine the overall acculturation of FSS to Westville University. The more they acquired sufficient understanding of English, their overall coping mechanism to learn the curriculum was enhanced. Evidence of linguistic power relations in this study reveals the cascading effects of the continuity-displacement contradiction because stakeholders exercised dominance of their preferred language of communication. This practice resulted in exclusion when stakeholders engaged 
in their preferred languages in the ACOP resulting in hampered interaction. As suggested by Lave and Wenger (1991), the effects of linguistic power relations fizzled out with time because FSS and SAS maintained their self-esteem and space. We envisage the disappearance of linguistic power dynamics in the ACOP as tensions of linguistic preferences equilibrate in trickles within the community. Similarly, findings in this regard support the claims made by Ramburuth and Tani (2009) that in a multicultural milieu issues of linguistic disparity abound.

In the COP framework members are seen as capable of "crossing boundaries" to other groups of intertwined COP with elapsed time and given space (Wenger, 1998, 126-133). The idea of members shifting positions otherwise referred to by Wenger as "crossing boundaries" is conceptualized in this study to imply the movement of FSS from one echelon of language (French) to the other (English). On the other hand, SAS and the other foreign students in the COP were supposed to cross their linguistic boundaries to attain an identity of using English to interact for learning to take place. Linguistic power dynamics in this fashion was found to limit the capacity of stakeholders "crossing boundaries" to attain a common identity for interaction that leads to learning to take place. Although stakeholders attempted to cross other boundaries in this study as suggested by Wenger (1998, 126-133), issues of linguistic power relations limited their fluidity in terms of crossing from one linguistic boundary to the other. Consequently, we agree with Wenger $(1998,126-133)$ that in COP members are capable of crossing from one boundary of practice to another. We add that the capacity of members to cross boundaries is dependent on how tenaciously they hold on to cultural tenets and their ease of intermingling with other cultures via an agreed medium of interaction. The FSS and SAS did not acquire the necessary dimensions of intercultural competence to manage their linguistic differences. FSS and SAS remained at both ends of a continuum and could not reach any reasonable consensus as far as linguistic adjustment was concerned. They interacted with one another in English when they were compelled by the curriculum during group work activities. Their interactions were short-lived because they returned into their linguistic enclaves after group work activities which were insufficient to make them negotiate the brisk transition which could translate into eloquence in English. We argue that the acquisition of intercultural competence is a prerequisite to extinguishing issues pertaining to linguistic power relations.

\subsection{Inflationary power relations}

This was brought about as a result of the declining affordability of FSS' parents in terms of funding the education of their children at Westville University. Narratives from FSS advisors constitute the basis for making this claim. A notable challenge to the influx of FSS to Westville University in the near future was the yearly escalation of fees and living expenses in South Africa. This was one of the issues confronting the FSS advisors when they went for marketing every year in French-speaking countries. Although the issue of yearly escalation in fees was common to all the students at Westville University, it was severe on the FSS because of the exchange rate of their currency to the Dollar, which was becoming unaffordable to parents. The five student advisors interviewed in this study unanimously agreed that the escalating tuition cost was posing a challenge to the influx of FSS into SA. Rosette (a student advisor) said that the registration fee at Westville University, which amounted to R800 (about USD 80), was enough to pay the tuition for the whole year in a Gabonese public University. Therefore, yearly escalation of fees and living expenses were challenges confronting the sustainability of FSS at Westville University. We present Rosette's expression on the issue of fees and living expenses to portray the concerns of parents and guardians of the FSS:

There is a rise in inflation in South Africa, so this is one other challenge to students. The cost of living is getting higher, and every year the tuition increases. The registration fee paid by French speakers at this school is enough to pay the registration fees of the Gabonese students back home for a year. From 2013, the Gabonese government will no longer send students here on sponsorship because they say it is too expensive.

\section{To support Rosette's narrative, Ms. Sammy's concerns about escalating fees are also presented:}

\footnotetext{
Last year there were quite a number of French-speaking students at this institution. In 2013 the number has reduced due to inflation. Our government in Gabon no longer sponsors students because it is becoming too expensive. Only rich parents bring their children here. The other challenge is the yearly escalation of $10 \%$ in fees. When will this escalation stop?
}

The anxieties of FSS advisors portray the undertone of inflation as challenges confronting the sustainability of FSS' academic pursuits at Westville University. Inflationary power relations depicted a stance of struggle to sustain the representation of FSS at Westville University. The rise in inflation typifies the power dynamics and the implication on the continuity of FSS' representation at Westville University. Utilising the continuity-displacement contradiction dynamics, we 
assert that inflationary power relations emerge when the financial capacity to educate students in an academic institution diminishes as a result of rising fees and living expenses. The preponderant show of financial dilemma stipulating that only those who are capable of measuring up to the standard of paying fees, despite the yearly escalation is a show of power a similitude of the neoliberal agenda where only the rich are capable of educating their children. This show of power gradually excluded FSS from the terrain of affordability. This drift tends to limit the sociocultural diversity in a so-called internationalized academic environment. This makes the suggestion of Lave and Wenger about the continuitydisplacement contradiction (1991, 115-116), that issues of power relations are subdued relevant in this study. The assertion of Lave and Wenger in this regard is relevant because the dynamics of the inability to cope with escalating fees fizzles out when those affected relinquish their willingness to pay by looking for alternative routes to obtain education. One of the student advisor laments, "We meet with resistance when we market this institution to parents outside this country because they are now beginning to see countries like Ghana as an alternative to educate their children because it is cheaper to study there." We argue that inflationary power relations flourished at Westville University. We argue that inflationary power relations are triggered by financial power relations in an ACOP.

\title{
5.4 Workplace power relations
}

A very challenging experience negotiated by the FSS was in terms of their accommodation on getting to Westville University. Each year the school residence gets filled up because of its proximity to the campus. Eventually the FSS who had been informed they would be granted accommodation before they came to Westville University were confronted by uncertainties because of the limited space in the hostels. The FSS advisors on the other hand were incapacitated because they usually promised the FSS that there was sufficient accommodation. The dilemma at such times on the part of student advisors was to get a safe accommodation for the FSS elsewhere. The management of Westville University made the FSS advisors sign undertakings to market the school accommodation. If they were caught looking for accommodation for any of the FSS outside of the school facilities they could be sacked. The FSS on the other hand are left with the feeling that they had been deceived because they were promised accommodation before they consented to coming to study in South Africa. The situation of the FSS advisors was a predicament because they wanted to please parents and at the same time the school authorities. As suggested by Lave and Wenger (1991), such power relations fizzled out with time because FSS and student advisors had to bow to the stipulations at Westville University. However, it did not help the marketing strategy of the French-speaking student advisors to recruit more students from Frenchspeaking countries. Rosette laments in response to the question put to her on the challenges student advisors faced in an attempt to market Westville University to the FSS:

\begin{abstract}
Many of the French-speaking students were turned away and you can imagine coming from a foreign country and getting here, they are not able to get accommodation. Also as student advisors we are not allowed to find alternative accommodation for them. And we are the ones who bring them here, so you see the position we are in now? If they get to know that I look for accommodation for them, I could be sacked. We are the ones who go to them in their countries and we convince them to come to study in South Africa so you can imagine what we face.
\end{abstract}

Rosette's argument is supported by another FSS advisor's dilemma. Mr Fletcher's concerns with respect to accommodating FSS are provided, an indication of workplace power relations:

\begin{abstract}
We help French-speaking students to integrate easily into this school because when they come here they need to be comfortable. When they know there are people here who speak French it helps them to easily stabilize. Secondly, we are here because when they struggle they can easily come to us and we liaise with them so as to get their problems solved. However, the issue of accommodation is a challenge. We promise them they would be accommodated before arriving here but we disappoint them because the residence is always full and we are not allowed to assist them to look for accommodation elsewhere because the management says we must market the residence.
\end{abstract}

These were only two cases of workplace power relations from the perspectives of FSS advisors out of the five cases obtained in this study. The fact that FSS advisors identified the requirement to maintain their promises to FSS and they were denied by the management of Westville University was indicative of the power struggle between the stakeholders. According to Billett (2006) a workplace has a curriculum which has to be followed by stakeholders. The workplace curriculum is seen in this study as the inauguration of power dynamics which are aimed at fostering the continuity of the workplace. Workplace curriculum, according to Billett (2006) is an array of formal and informal stipulations on a daily basis that advance the workplace. We agree with Billett (2006) that the workplace curriculum is a 
stipulation of official and unofficial stipulations, aimed at propagating the interest of the workplace. According to Hodkinson and Hodkinson $(2003,6)$, Lave and Wenger are clear-cut on the connotation of power dynamics in creating admission to workplace learning. Nevertheless, the discussion of Lave and Wenger (1991) on issues of power relations was not in detail. We agree with the observation of Hodkinson and Hodkinson and contribute that the learning available to stakeholders in terms of power relations at the workplace is that those vested with authority use it to the fullest no matter how the other lower ranking stakeholders may feel. The FSS advisors thus vacillated between the dynamics of continuity with their work as they confronted issues of displacement by the attempt to fulfil their promise to the FSS at Westville University by virtue of the contradictions they experienced from Westville University management.

It is the opinion of Lave and Wenger $(199,116)$ that power dynamics between experts and novices ensue in the course of daily routines in the community, as was seen between the student advisors and management at Westville University. However, joint involvement is the phase through which old timers and new, recognised and unrecognised, the reputable and the expectant, settle their dissimilarities and notice their shared aims, divulge their fright of one another, and ascertain their usefulness of one another. This notion was likely responsible for the continued silence of student advisors at Westville University in an attempt to keep their jobs and to meet specified sales target. Participants in the community coerced the achievement of the other's goal in the same way that it is significant to it. For example, management was found to compel the achievement of student advisors to ensure that FSS registered at Westville University at the detriment of student advisors' integrity. Disagreement between the management of Westville University and student advisors was encountered and negotiated via a joint daily exercise through which opposing views and communal interests interacted. As proposed by Lave and Wenger power relations between the management of Westville University and student advisors were subdued, although not snuffed through the contrasts of power dynamics between the very experienced (management of Westville University) and beginners (FSS advisors). As an avenue through which the associated power dynamics are balanced out in practice, the LPP model surpasses the course of learning among new entrants. The LPP model is seen as a mutually symbiotic enterprise between people and profession, an assertion which corroborates the tenet proposed by Lave and Wenger $(1991,116)$.

\section{Conclusion and Recommendations}

It was noticed that there was no institutionalized measure to deliberately attach FSS to SAS as was the case in other studies (e.g. Quintrell and Westwood, 1994). It is recommended that peer-pairing initiatives should be practised at Westville University to enhance the possibility of interaction and lower chances of linguistic power relations. Inflationary power relations excluded students without adequate financial capacity from being educated. It was an encounter that gradually reduced the number of foreign students' representation at Westville University. Workplace power relations became the centre stage between stakeholders at Westville University because of the struggle to maintain business in a dimension that fostered continuity. Management at Westville University preferred to market the residential apartments at the detriment of FSS' satisfaction, although demand far outweighed supply. It was an attempt to foster continuity in business without the option of displacement as far as the intake of FSS was concerned. Our underlying assumption at the outset, that the curriculum experiences of FSS are challenged by power relations has been shown to be relevant. We recommend that stakeholders should critically engage in communication on a regular basis to alleviate the concerns of FSS in terms of the continuity-displacement contradictions so that an agreement can be made to address their concerns. In summary, financial power relations, linguistic/cultural power relations, workplace power relations and inflationary power relations are threats to international students' representation and the quest to internationalize the campus environments of higher education institutions.

\section{References}

Bennett, J., M. J. Bennett and W. Allen. 2003. Developing intercultural competence in the language classroom. In Culture at the core: Perspectives on culture in second language learning, eds. D. L. Lange and R. M. Paige, 237-270. Greenwich, CN: Information Age Publishing.

Billett, S. 2006. Constituting the workplace curriculum. Journal of Curriculum Studies 38(1): 31-48.

Braun, V. and V. Clarke. 2006. Using thematic analysis in psychology. Qualitative Research in Psychology 3: 77-101.

Burden, R. 1997. Research in the real world: An evaluation model for use by applied psychologists. Educational Psychology in Practice 13(1): 13-29.

Chow, H. P. H. 2006. Vietnamese-Canadian University Students in Regina: Sociocultural and Educational Adaptation. Canadian Ethnic Studies 38(2): 104-112.

Denzin, N. K. and Y. S. Lincoln. 2005. The discipline and practice of qualitative research. In Sage handbook of qualitative research, eds. 
N. K. Denzin and Y. S. Lincoln, 1-32. Thousand Oaks, CA: Sage.

Durning, S. J. and Artino, A.R. (2011). Situativity theory: A perspective on how participants and the environment can interact: AMEE Guide, 52(33): 188-199.

Hodkinson, P. and H. Hodkinson. 2003. Individuals, communities of practice and the policy context: School teachers' learning in their workplace. Studies in Continuing Education 25(1): 3-21.

Lave, J. and E. Wenger. 1991. Situated learning: Legitimate peripheral participation. Cambridge, UK: Cambridge University Press.

Lave, J. 1997. The culture of acquisition and the practice of understanding. In Situated cognition: Social, semiotic and psychological perspectives, eds. D. Kirshner and J. A. Whitson, 17-35. Mahwah, NJ: Lawrence Erlbaum Associates.

Mayring, P. 2000. Qualitative content analysis. Forum for Qualitative Research: Qualitative Social Research 1(2). Available at: http://qualitative-research.net/fqs/fqs-e/2-00inhalt-e.htm. (accessed 20 February 2009).

Miles, M. B. and A. M. Huberman. 1994. Qualitative data analysis: An expanded sourcebook. Second edition. London and Thousand Oaks: Sage.

Otten, M. 2009. Academicus Interculturalis? Negotiating interculturality in academic communities of practice. Intercultural Education 20(5): 407-417.

Quintrell, N. and M. Westwood. 1994. The influence of a peer-pairing programme on international students' first year experience and use of student services. Higher Education Research and Development 13(1): 49-57.

Ramburuth, P. and M. Tani. 2009. The impact of culture on learning: Exploring student perceptions. Multicultural Education \& Technology Journal 3(3): 182-195.

Sandelowski, M. 2000. Focus on research methods. Whatever happened to qualitative description? Research in Nursing and Health 23 : 334-340.

Smart D., S. Volet, and G. Ang. 2000. Fostering social cohesion in universities: Bridging the cultural divide. Canberra: Australian Education International.

Tanaka, T., J. Takai, T. Kohyama, T. Fujihara, and H. Minami. 1997. Effects of social networks on cross-cultural adjustment. Japanese Psychological Research 39(1): 12

Trice, A. G. 2004. Mixing it up: International graduate students' social interactions with American students. Journal of College Student Development 45(6): 671-87.

Vandeyar, S. 2010. Shifting selves: Constructing and negotiating academic identities. South African Journal of Higher Education 24(6): 914-934.

Wenger, E. 1998. Communities of practice: learning, meaning and identity. New York: Cambridge University Press.

Yeh, C. J., Y. Okubo, P. W. Ma, M. Shea, D. Ou, and S. T. Pituc. 2008. Chinese immigrant high school students' cultural interactions, acculturation, family obligations, language use, and social support. Adolescence 43(172): 775-790. 